\section{The Short-Range Resistive Wall Wakefields}

Karl L.F. Bane and Matthew Sands

Stanford Linear Accelerator Center

Stanford University, Stanford, California 94309

\begin{abstract}
In an accelerator when the bunch length becomes comparable to a characteristic distance $s_{0}$, one which depends on the radius and the conductivity of the beam tube and in typical structures is on the order of tens of microns, the usual formulas for the resistive wall wakefield do not apply. In this report we derive the short-range resistive wall wakefields of an ultra-relativistic point particle in a metallic, cylindrical tube, both for a model in which the wall conductivity is taken to be independent of frequency and for one in which a frequency. dependence is included. On this scale the wakefield is found to be dominated by a damped, high frequency resonator component. For the case of constant conductivity the resonant frequency is given by $\omega=\sqrt{3} \mathrm{c} / \mathrm{s}_{0}$ and the $Q$-factor equals $\sqrt{3} / 2$. We provide a physical model to explain these results. For the case of a frequency dependent conductivity the resonator parameters depend also on the relaxation time of the metal $\tau$. For $c \tau / s_{0} \gtrsim 0.5$ the frequency $\omega \approx \sqrt{2 \omega_{p} c / b}$, with $\omega_{p}$ the plasma frequency of the free electrons in the metal and $b$ the tube radius, and the $1 / e$ damping time becomes $4 \tau$. Finally, we calculate the wakefield and loss factor of a short Gaussian bunch.
\end{abstract}

\section{INTRODUCTION}

In some recent accelerator designs electron bunches with high peak currents and very short bunch lengths (so-called "micro-bunches") are envisioned. For example, in one version of the Linac Coherent Light Source (LCLS) project at SLAC, a project that intends to use the SLAC linac as a driver for a very short wave-length FEL, the peak current is $5 \mathrm{kA}$ and the longitudinal bunch shape is non-Gaussian (somewhat rectangular) with an rms length of $20 \mu \mathrm{m}$ (1). Under such conditions the normally weak resistive wall wakefields of a beam tube can be strong, comparable in strength, for example, to those of accelerating cavities of the same beam tube radius. In addition, for such short bunches the usual formulas for the resistive wall wakefields no longer apply. The object of this report is to calculate the resistive wall wakefields that are applicable over such short distances.

Consider an ultra-relativistic bunch traversing a cylindrical, metallic tube. The metal is made of a good conductor; its surface is smooth. In this paper Work Supported by the Department of Energy, contact DE-AC03-76SF00515.

\section{DISTRIBUTION OF THIS DOCUMENT IS UNLIMITED}

Presented at Micro Bunches: A Workshop on the Production, Measurement and Applications of Short Bunches of Electrons and Positrons in Linacs and Storage Rings, Upton, L.X., New York, September 28-30, 1995 we are interested in the wakefield of a short bunch, by which we mean that the bunch length is comparable to the characteristic distance (see, e.g. Ref. 2)

$$
s_{0}=\left(\frac{c b^{2}}{2 \pi \sigma}\right)^{1 / 3}
$$

with $c$ the speed of light, $b$ the tube radius, and $\sigma$ the conductivity of the metallic walls. For copper at room temperature $\sigma=5.8 \times 10^{17} \mathrm{~s}^{-1}$. For a copper tube with $b=1 \mathrm{~cm}, s_{0}=20 \mu \mathrm{m}$. Although in accelerators the bunch length is normally much larger than this value the above LCLS example falls within the short bunch category if the tube radius is comparable to $1 \mathrm{~cm}$. Note also that, for a given bunch length, as we increase $b$ we will also increase $s_{0}$. For the above example, if we increase $b$ to $10 \mathrm{~cm}$ then $s_{0}$ becomes $93 \mu \mathrm{m}$.

The conductivity of a metal is not independent of frequency. In the presence of oscillating fields Ohm's law becomes $\tilde{\mathbf{J}}=\bar{\sigma} \tilde{\mathbf{E}}$ with (3)

$$
\bar{\sigma}=\frac{\sigma}{1-i \omega \tau},
$$

with $\omega$ the frequency of oscillation and $\tau$ the relaxation time of the metal. We note that it is only at higher frequencies that $\bar{\sigma}$ (which we will call the ac conductivity) differs significantly from $\sigma$ (the dc conductivity). For copper at room temperature the relaxation time $\tau=2.7 \times 10^{-14} \mathrm{~s}$ or $\mathrm{cr}=8.1 \mu \mathrm{m}$. [Note that for $\mathrm{Ag}, \tau=4.0 \times 10^{-14} \mathrm{~s}$; for $\mathrm{Al}, \tau=0.8 \times 10^{-14} \mathrm{~s}$. Note also that $\bar{v} \tau=\ell$, with $\bar{v}$ the average velocity of the free electrons in the metal and $\ell$ the mean free path between collisions.] Let us introduce the dimensionless relaxation factor $\Gamma=c \tau / s_{0}$. For a $1 \mathrm{~cm}$ copper tube $\Gamma=0.4$. Therefore, for this example, for frequencies $\omega \sim c / s_{0}$, the second term in the denominator of Eq. (2), in amplitude, becomes comparable to the first term; we might therefore expect the short range ac wakefield to be noticeably different from the short range dc wake.

Down to what length scale do we expect that our calculations are valid? An important assumption in all our calculations is that Ohm's law applies in the metallic walls. One effect, the anomalous skin effect, describes a break-down of Ohm's law when the mean free path $\ell$ becomes large. However, this effect is normally important only at low temperatures (4) and will not be considered here. In our calculations we will drop a very high frequency term arising from the displacement current, a term which only becomes important when $\omega \approx \sqrt{4 \pi \sigma / \tau}=\omega_{p}$, with $\omega_{p}$ the plasma frequency of the free electrons in the

* All physical properties of metals presented in this paper can be found in Ref. 3 . 
metal. For a copper tube at room temperature the equivalent distance is $c / \omega=$ $0.02 \mu \mathrm{m}$. However, we expect that at submicron distances, before reaching this level, quantum mechanical effects will invalidate the classical treatment given here [For a more detailed discussion on the electrical behavior of real metals at high frequencies see, for example, Ashcroft and Mermin (3)]. Finally, our calculations in this paper assume a beam energy of infinity, resulting in an impedance that reaches to infinite frequencies. For beams of finite energy, however, the impedance will be cut off at frequencies $\omega \sim \gamma c / b$, with $\gamma$ the beam energy. For a $1 \mathrm{GeV}$ beam in a $1 \mathrm{~cm}$ tube this corresponds to a cut-off distance of $c / \omega=5 \mu \mathrm{m}$.

The long-range resistive wall wakefields have been well understood for many years (for early papers see, for example, Refs. 5-9). In A. Chao's book on collective beam instabilities (2) the author finds the long-range resistive wall wakefields due to a point charge moving at the speed of light in a cylindrical, metallic tube by field matching. We will employ Chao's approach to find the short-range resistive wall wakefields of a point charge. Note that the short-range resistive wall wakefields were first obtained for a boundary consisting of two parallel plates by Henke and Napoly (10) and then in a circular tube (as here) by Henry and Napoly (11), where the authors treated the dc conductivity part of this report and obtained some of the same numerical results. Note also that this paper is essentially the same as Ref. 12, from which the dc conductivity results have been reproduced in Chao's book. This report appears here because: (a) it seems that the short-range resistive wall wakefield may be important for future accelerator projects employing "micro-bunches", (b) Ref. 12 is not widely available, and (c) this report differs significantly from Henry and Napoly's in: (i) our approach is different, (ii) we give the results in a different form, (iii) we include a physical interpretation of our results, and (iv) we include results for an ac conductivity.

\section{The Long-Range Wakefields: Chao's Calculation}

We begin by describing Chao's resistive wall calculation. Consider an infinitely long, cylindrical tube whose axis coincides with the $z$-axis. The tube is made of a smooth metal with a high conductivity $\sigma$, a constant. The metallic walls begin at $r=b$ and are not thin. Now consider a point charge $q$ moving from $z=+\infty$ to $z=-\infty$ along the $z$-axis at the speed of light $c$, and which at time $t=0$ is at location $z=0$. All the fields left by the charge depend on $z$ and $t$ only in the combination $z-c t$. Let the longitudinal position variable to be $s=-z+c t$, with negative values of $s$ located in front of the driving charge.
The longitudinal impedance per unit length $Z$ is given by $-1 / q$ times the Fourier transform of the longitudinal electric field, $\tilde{E}_{z}$, i.e.

$$
Z(k)=-\frac{1}{q c} \int_{-\infty}^{\infty} d s E_{z}(s) e^{i k s}
$$

In Chao's report the longitudinal impedance is found in the following manner: (i) Maxwell's Equations are written in cylindrical coordinates in the tube region, (ii) the equations are Fourier transformed, (iii) these two steps are repeated for the fields in the metallic region, and (iv) $\tilde{E}_{z}$ and $\tilde{B}_{\phi}$ for the two regions are matched at $r=b$ to give the coefficent, which is the impedance. The result is

$$
Z=\frac{2}{c b}\left[\left(\frac{\lambda}{k}+\frac{k}{\lambda}\right)\left(1+\frac{i}{2 \lambda b}\right)-\frac{i k b}{2}\right]^{-1}
$$

with

$$
\lambda^{2}=\frac{4 \pi \sigma i k}{c}
$$

Since the fields in the walls vary as $e^{i \lambda r}$ the root of $\lambda^{2}$ that is in the upper half of the complex plane is chosen:

$$
\lambda=\sqrt{\frac{2 \pi \sigma|k|}{c}}[i+\operatorname{sgn}(k)],
$$

where $\operatorname{sgn}(k)$ is the sign of $k$. Dropping the very low frequency term $[i /(2 \lambda b)]$ and the very high frequency term $(k / \lambda)$, Eq. (4) can be approximated by

$$
Z=\frac{2}{c b}\left[\frac{\lambda}{k}-\frac{i k b}{2}\right]^{-1}
$$

In terms of the characteristic distance $s_{0}$ and the dimensionless frequency $\kappa=k s_{0}$ Eq. (7) can be written as

$$
Z=\frac{2 s_{0}}{c b^{2}}\left[\frac{i \operatorname{sgn}(\kappa)+1}{|\kappa|^{1 / 2}}-\frac{i \kappa}{2}\right]^{-1}
$$

As long as one's interest is limited to $s$ large compared to $s_{0}$ the first term in the brackets of Eq. (8) dominates, and the second term can be dropped. 
Chao makes this approximation, performs the inverse Fourier transform of the result, and obtains

$$
E_{z}=\frac{q}{2 \pi b} \sqrt{\frac{c}{\sigma}} \frac{1}{s^{3 / 2}}=\frac{q}{\sqrt{2 \pi} b^{2}}\left(\frac{s_{0}}{s}\right)^{3 / 2}
$$

Note that the sign is such that the long-range resistive wall wakefield will increase the energy of a test particle. Note also that in units of $q / b^{2}$ the long range electric field is usually very small. Finally, keeping the second term in the brackets of Eq. (8) and dropping the first, and performing the inverse Fourier transform, Chao finds that $E_{z}(0)=-4 q / b^{2}$, a result which he also obtains from energy conservation considerations.

\section{THE SHORT-RANGE WAKEFIELDS ASSUMING A CONSTANT CONDUCTIVITY}

To find the short range wakefield we take the inverse Fourier transform of $Z$ as given by. Eq. (8), but now keeping both terms in the equation. The real and imaginary parts of $Z$ are plotted in Fig. 1. From the plot the impedance appears to consist primarily of a broad resonance. The frequency for which the two terms of the equation, in absolute value, are equal is $\kappa=2$. We note that this frequency is near the peak position of the resonance.

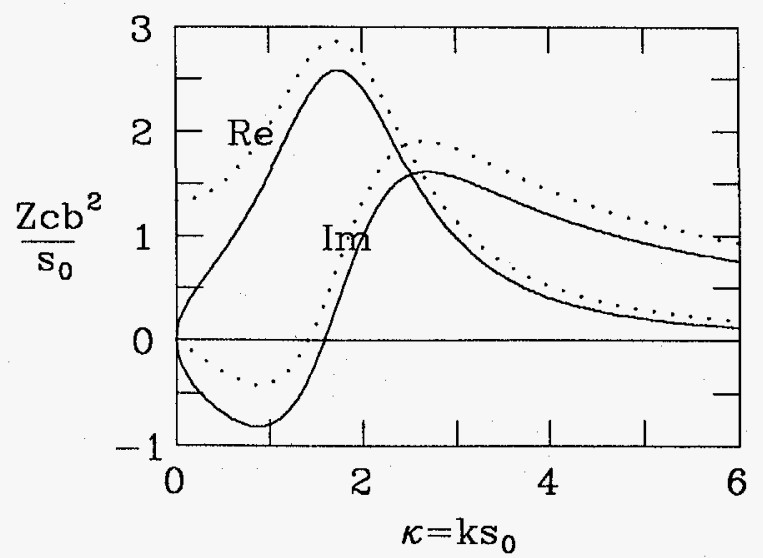

FIGURE 1. The real and imaginary parts of $Z$ as functions of $\kappa=k s_{0}$, as given by $\mathrm{Eq} .(8)$. The dots give the Fourier transform of the resonance term of $E_{z}$ (to be discussed below).
To compute the inverse Fourier transform of the impedance we first replace $Z$ by an analytic function $\bar{Z}$ that equals $Z$ on the real axis. In this case

$$
\bar{Z}=\frac{2 s_{0}}{c b^{2}}\left[\frac{i+1}{\kappa^{1 / 2}}-\frac{i \kappa}{2}\right]^{-1}
$$

In this equation we interpret $\kappa$ to be a complex variable. Our complex plane has a branch cut on the negative $\operatorname{Im}(\kappa)$ axis; we confine ourselves to the Riemann sheet $-\pi / 2<\theta<3 \pi / 2$. The electric field is then given by

$$
E_{z}(s)=\frac{q}{2 \pi s_{0}} \int_{-\infty}^{\infty} d \kappa \bar{Z}(\kappa) e^{-i \kappa s / s_{0}}
$$

with the integral taken along the $\operatorname{Re}(\kappa)$ axis. Now consider the closed contour shown in Fig. 2. The integral around the path equals $(-2 \pi i)$ times the sum of the residues at the poles. The function $\bar{Z}$ has two simple poles in the lower half of the contour plot, one at $\kappa=\sqrt{3}-i$, the other at $\kappa=-\sqrt{3}-i$. If we deform the contour by increasing the radius of the arcs to infinity, then the integrals over the arcs become zero. Therefore, $E_{z}(s)$ equals $(2 \pi i)$ times the sum of the residues, minus the sum of the integral over the path $C_{1}$ plus that over the path $C_{2}$. Performing the calculations we obtain

$$
E_{z}(s)=-\frac{16 q}{b^{2}}\left(\frac{1}{3} e^{-s / s_{0}} \cos \frac{\sqrt{3} s}{s_{0}}-\frac{\sqrt{2}}{\pi} \int_{0}^{\infty} \frac{d x x^{2} e^{-x^{2} s / s_{0}}}{x^{6}+8}\right)
$$

The first term in Eq. (12) gives the contributions of the residues, the second term those of the integrals over the paths $C_{1}$ and $C_{2}$.

The solution is plotted in Fig. 3 . We see that $E_{z}$ begins with a negative value, changes sign three times, and remains positive beyond $s=4.2 s_{0}$. Note that a similar oscillatory behavior has been found for the short-range resistive wall wakefield of a beam between two parallel plates (10). From Eq. (12) we note that $E_{z}$ consists of two terms. The first term represents a high frequency, damped resonator, with wave number $k_{r}=\sqrt{3} / s_{0}$ and damping factor $a_{r}=$ $1 / s_{0}$. At times it is convenient to describe the damping in terms of the quality factor $Q$, defined as $Q=k_{r} /\left(2 a_{r}\right)$; in this case $Q=\sqrt{3} / 2$. The resonator contribution to $E_{z}$ is shown by the dashes in Fig. 3. We see that over the short range the resonator term alone well approximates the entire wakefield. 


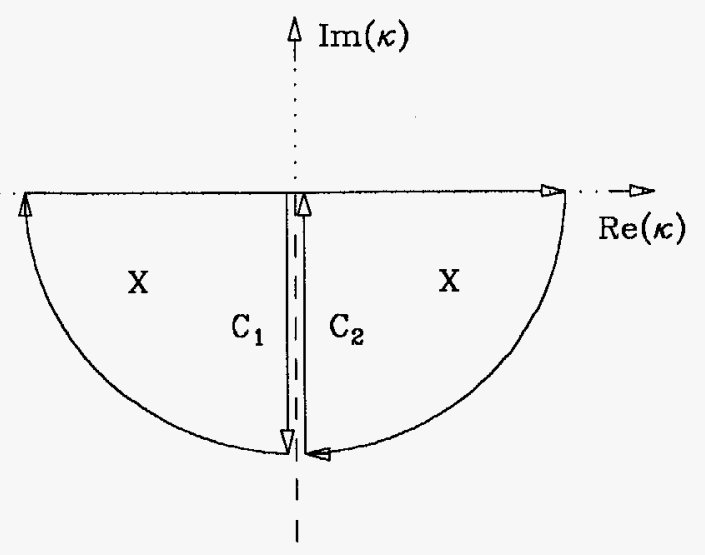

FIGURE 2. The contour of integration for this problem. The dashed line shows the branch cut, the X's indicate the poles.

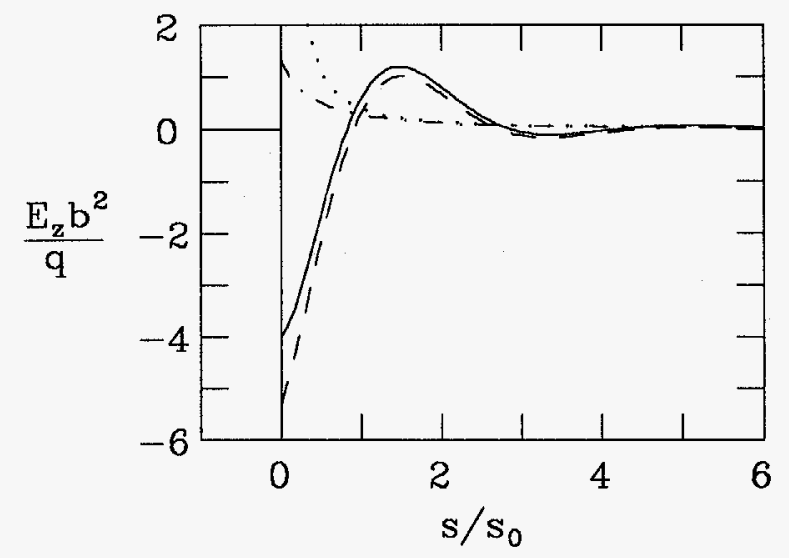

FIGURE 3. The short-range longitudinal resistive wall wakefield (the solid curve). Also shown are the oscillator component (the dashes), the diffusion term (the dotdashes), and the long range solution of $E_{z}$ [see Eq. (9)] (the dots).

This can similarly be seen in the frequency domain. If we Fourier transform $-1 / q$ times the resonator term of Eq. (12) we obtain

$$
Z_{r}=-\frac{16 q s_{0}}{3 b^{2}} \frac{1+i \kappa}{4+2 i \kappa-\kappa^{2}}
$$

(see the dotted curves in the impedance plot, Fig. 1). We see that this contribution well approximates the entire impedance, except at low frequencies.

The second term in Eq. (12), which is positive definite and monotonically decreasing, we will call the diffusion term (see the dotdash curve in Fig. 3 ). For large $s / s_{0}$ it is this term that dominates, in which range it is well approximated by $\mathrm{Eq} .(9)$, the normal, long range result (the dotted curve in Fig. 3). The entire beam/wall interaction can be roughly thought of occurring in two parts. First the beam loses energy to the high frequency resonator and then, on a longer time scale, this energy diffuses into and is absorbed by the walls. Finally, note that since

$$
\int_{0}^{\infty} \frac{d x x^{2}}{x^{6}+8}=\frac{\pi}{12 \sqrt{2}},
$$

$E_{z}\left(0^{+}\right)=-4 q / b^{2}$, as is required

\section{A Simple Model of the Damped Oscillation}

How can we understand the high frequency, damped oscillating character of the short range wakefield? In this section we describe a physical mechanism that can account for such a high frequency resonance. Consider a wave propagating down a tube. It is well known that if the tube is perfectly conducting then a relativistic particle cannot interact with the wave. That is, in the dispersion diagram the speed of light line never crosses the dispersion curve. If, however, the tube has a finite conductivity then the dispersion curve will be modified, and the two curves will cross. The position of crossing gives the resonant frequency. If the conductivity is high, as in the case of good conductors, the dispersion curve will be only slightly modified, and we would therefore expect the resonant frequency also to be high.

We use a perturbation method to estimate the resonant frequency. A propagating wave in a perfectly conducting tube has a dispersion relation given by

$$
k_{\lambda}^{(0)}=\frac{\omega}{c} \sqrt{1-\left(\frac{\omega_{\lambda}}{\omega}\right)^{2}},
$$

with $k_{\lambda}^{(0)}$ the axial wave number and $\omega_{\lambda}$ the cut-off frequency of the mode. A particle can only interact with a wave with which it is synchronous; if the particle moves at the speed of light then the wave number needs to equal $\omega / c$. Since $k_{\lambda}^{(0)}$ is always less than $\omega / c$ we see that such a particle does not interact 
with a wave in a perfectly conducting tube. For a tube with a finite but high conductivity let the wave number be approximated by

$$
k_{\lambda} \approx k_{\lambda}^{(0)}+\alpha_{\lambda}+i \beta_{\lambda}
$$

with $\alpha$ and $\beta$ small real numbers. By the method of perturbation of boundary conditions it is shown in Jackson (13), Sections 8.5-8.6, that $\alpha_{\lambda}=\beta_{\lambda}$ and that for TM modes in a cylindrical tube

$$
\alpha_{\lambda}=\frac{c}{4 \pi \sigma \delta_{\lambda} b}\left(\frac{\omega}{\omega_{\lambda}}\right)^{1 / 2}\left[1-\left(\frac{\omega_{\lambda}}{\omega}\right)^{2}\right]^{-1 / 2},
$$

with $\delta_{\lambda}=c / \sqrt{2 \pi \omega_{\lambda} \sigma}$ the skin depth at cut-off. In Eq. (17) we have set both the dielectric constant and the permeability to 1 . Note that $\alpha_{\lambda}$ is positive, and therefore a relativistic particle can now be synchronous with the wave. Setting $\omega / c=k_{\lambda}$, and assuming that $\omega / \omega_{\lambda} \gg 1$, we find that

$$
\frac{\omega}{c}=k_{\lambda}^{(0)}+\alpha_{\lambda} \approx \frac{\omega}{c}\left[1-\frac{1}{2}\left(\frac{\omega_{\lambda}}{\omega}\right)^{2}\right]+\frac{1}{2 b} \sqrt{\frac{\omega}{2 \pi \sigma}}
$$

The solution of this equation is $\omega=\left(2 \pi \sigma b^{2} \omega_{\lambda}^{4} / c^{2}\right)^{1 / 3}$. Let us assume that the beam only interacts with the first TM mode, for which $\omega_{\lambda} b / c \approx 2.4$. Then our solution becomes $\omega / c=3.2 / s_{0}$, which is a factor of 1.8 larger than that given in the previous section. Given the approximate nature of this calculation the agreement is quite good.

Note that the relative size of the damping term $\beta_{\lambda} c / \omega=\left(\omega_{\lambda} / \omega\right)^{2} / 2=$ $0.3\left(s_{0} / b\right)^{2}$ is small, as is necessary for the perturbation calculation to be valid. However, this observation does not appear to be in agreement with the low $Q$-factor that was found in the previous section. To explain this we suggest that the low $Q$-factor does not result from damping of the waves, but rather from the spread of frequencies in the waves excited. To aid in visualizing this we display schematically the dispersion diagram for this problem in Fig. 4. Two, rather than one, lines are plotted; their separation indicates the width of the resonance $\delta k_{\lambda} \approx \beta_{\lambda} \ll k_{\lambda}$. The dashed line, with a slope of $1 / c$, represents the speed of light line. Let us take $\delta \omega$, the frequency interval for which the light line is between the dispersion curves, to indicate the effective width of the resonance. Since the crossing angle between the dispersion curves and the light line is very small we see that $\delta \omega / \omega$ will be much larger than $\beta_{\lambda} / k_{\lambda}$.

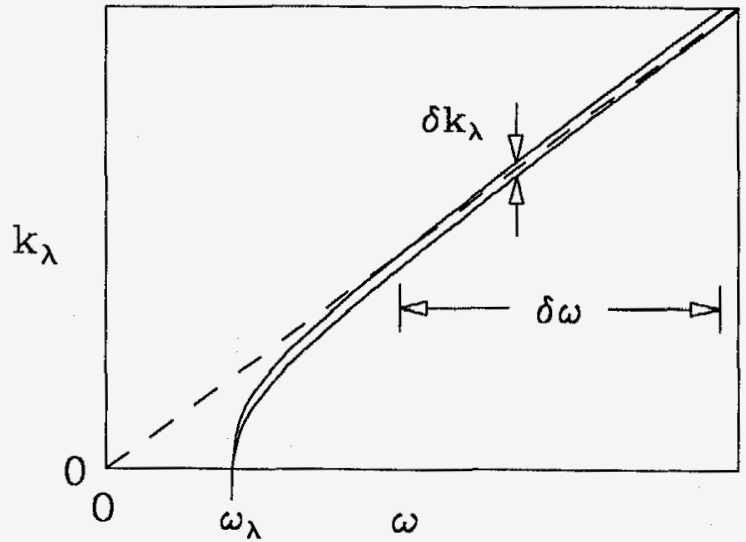

FIGURE 4. A sketch of one band of the dispersion curve for a resistive tube. The light line is given by the dashed line.

From the plot we see that

$$
\frac{\delta \omega}{\delta k_{\lambda}}=\left(\frac{d k_{\lambda}}{d \omega}-\frac{1}{c}\right)^{-1}
$$

with $d k_{\lambda} / d \omega$ the slope of the dispersion curve at resonance. But Eq. (18) gives

$$
\frac{d k_{\lambda}}{d \omega}=\frac{1}{c}+\frac{3 \beta_{\lambda}}{2 \omega}
$$

Thus by setting $\delta k_{\lambda}=\beta_{\lambda}$ we find that the effective $Q$-factor $\delta \omega / \omega \approx 2 / 3$, in rough agreement with the exact result $(\sqrt{3} / 2)$ found in the previous section.

\section{Higher Multipoles}

For higher azimuthal mode number $m$ Chao finds that in the tube region

$$
\tilde{E}_{z}^{(m)}=q Z^{(m)} r^{m}
$$

with

$$
Z^{(m)}=\frac{4 I_{m}}{q c b^{2 m+1}}\left(\frac{\lambda}{k}-\frac{i k b}{m+1}\right)^{-1} \quad m>0
$$


with $I_{m}$ the $\mathrm{m}^{\text {th }}$ multipole moment. By repeating the procedure used in calculating the monopole short range results we find $E_{z}^{(m)}$. The results (now valid for all $m$ ) is

$$
E_{z}^{(m)}=\frac{2 I_{m}}{\left(1+\delta_{m 0}\right)} \frac{r^{m}}{b^{2 m+1}} \bar{E}_{z}^{(m)}
$$

with $\delta_{m 0}$ the Kronecker delta function, and with

$$
\bar{E}_{z}^{(m)}(s)=-16 \gamma\left(\frac{1}{3} e^{-\gamma^{2 / 3} s / s_{0}} \cos \frac{\sqrt{3} \gamma^{2 / 3} s}{s_{0}}-\frac{\sqrt{2} \gamma}{\pi} \int_{0}^{\infty} \frac{d x x^{2} e^{-x^{2} s / s_{0}}}{x^{6}+8 \gamma^{2}}\right) .
$$

In Eq. (24) $\gamma=1$ when $m=0$ and $\gamma=(m+1) / 2$ when $m>0$. We note that for all $m$ the solution is very similar and consists of both a low $Q$ resonator and a positive definite, monotonically decreasing function. In addition we note that $\bar{E}_{z}$ is the same for the dipole $(m=1)$ mode as it was for the monopole mode. For the higher multipoles the resonator frequency increases whereas the $Q$-factor remains equal to $\sqrt{3} / 2$.

Given the longitudinal electric field of the higher multipoles we can find the transverse wakefields by a simple integration. This is a consequence of the Panofsky-Wenzel Theorem (14). For example, for the dipole mode, assuming the beam is displaced in the $x$-direction by an amount $x$, the transverse force on the test particle, divided by its charge, is given by

$$
F_{x}^{(1)}=-\frac{1}{x} \int_{0}^{s} d s E_{z}^{(1)}
$$

\section{THE SHORT-RANGE WAKEFIELDS INCLUDING AN AC CONDUCTIVITY}

\section{In the Limit of High $\Gamma$}

We begin with the high $\Gamma$ limit since in this regime the wakefield can be found analytically. The ac conductivity is given, in units of normalized frequency $\kappa=k s_{0}$, by

$$
\vec{\sigma}=\frac{\sigma}{1-i \kappa \Gamma}
$$

For high $\Gamma$, and for $\kappa$ not small, we can approximate the conductivity by

$$
\bar{\sigma} \approx \frac{\sigma}{\kappa \Gamma}\left(i+\frac{1}{\kappa \Gamma}\right)
$$

To include the ac conductivity in our calculations we replace $\sigma$ in Eq. (5) by $\bar{\sigma}$ as given in Eq. (27). Again taking the root of $\lambda^{2}$ to be in the upper half of the complex plane we find that

$$
\lambda=\frac{b}{s_{0}^{2}} \sqrt{\frac{2}{\Gamma}}\left[i+\frac{\operatorname{sgn}(\kappa)}{2 \kappa \Gamma}\right] .
$$

Next we substitute into Eq. (7) to obtain the impedance Z. Then, just as for the case of dc conductivity, we replace the impedance by an analytic function $\bar{Z}$ that is identical to $Z$ on the real axis. For this case we find that

$$
\bar{Z}=\frac{2 s_{0}}{c b^{2}}\left[\sqrt{\frac{2}{\Gamma}} \frac{1}{\kappa}\left(i+\frac{1}{2 \kappa \Gamma}\right)-\frac{i \kappa}{2}\right]^{-1} .
$$

The inverse Fourier transform of this function is

$$
\left.E_{z}(s)=-\frac{4 q}{b^{2}} e^{-s /\left(4 \Gamma s_{0}\right)} \cos \left[(8 / \Gamma)^{1 / 4} s / s_{0}\right)\right] .
$$

The result is a resonator with wave number $k_{r}=(8 / \Gamma)^{1 / 4} / s_{0}$ and damping factor $a_{r}=1 /(4 c \tau)$. The result can also be written in terms of the plasma frequency of the metal $\omega_{p}=\sqrt{4 \pi \sigma / \tau}$ :

$$
E_{z}(s)=-\frac{4 q}{b^{2}} e^{-s /(4 c \tau)} \cos \left[\sqrt{\frac{2 \omega_{p}}{b c}} s\right]
$$

Thus in the limit of high $\Gamma$ the resonant frequency depends on the ratio of the plasma frequency to the tube radius.

How high a value of $\Gamma$ can we expect to ever encounter? Recall that for a $1 \mathrm{~cm}$ copper tube at room temperature $\Gamma=0.4$. For a silver tube, this number increases by $50 \%$. We can also increase $\Gamma$ by cooling the metal; for copper, at $77^{\circ} \mathrm{K}, \Gamma=6.3$, but due to the anomolous skin effect the results given here are no longer valid. Finally, we increase $\Gamma$ by reducing the tube radius; for a copper tube at room temperature, with $b=2 \mathrm{~mm}, \Gamma$ becomes 1.5 .

\section{The General Case}

In order to calculate the ac resistive wall wakefield for arbitrary $\Gamma$ we replace the dc conductivity $\sigma$ in Eq. (5) by the general form of the ac conduc- 
tivity $\bar{\sigma}$ [see Eq. (26)]. Again taking the root of $\lambda^{2}$ to be in the upper half of the complex plane we find that

$$
\lambda=\frac{b}{s_{0}^{2}}|\kappa|^{1 / 2}\left(1+\kappa^{2} \Gamma^{2}\right)^{-1 / 4}\left[i \sqrt{1+t_{\lambda}}+\operatorname{sgn}(\kappa) \sqrt{1-t_{\lambda}}\right],
$$

with

$$
t_{\lambda}=\frac{|\kappa| \Gamma}{\sqrt{1+\kappa^{2} \Gamma^{2}}} .
$$

Substituting this expression for $\lambda$ into Eq. (7), we obtain the impedance $Z$. Fig. 5 gives the real and imaginary parts of $Z$ when $\Gamma=0.4$. For comparison the dotted curves give the dc results. We note that it is only for the higher frequencies - in this example for $\kappa \gtrsim 1$ - that the ac impedance differs significantly from the dc impedance. A major difference we see in the two impedances is that the resonance of the ac impedance is narrower, and therefore its $Q$-factor is larger, than it is for the dc impedance.

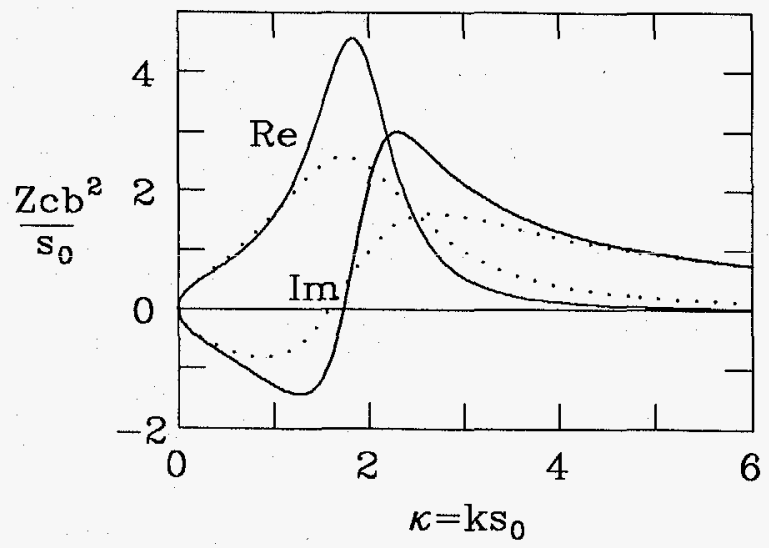

FIGURE 5. The real and imaginary parts of $Z$ for an ac conductivity with $\Gamma=0.4$. The dots give the dc conductivity results.

Let us try to analyze the impedance. From Eqs. (32),(33), it at first appears that the ac impedance is significantly more complicated than the dc impedance was. However, we expect that the-for our purposes-most important features of the impedance are no different than before: (i) the short range wake will still be dominated by a simple resonator term, (ii) this resonator term will be associated with two simple poles of an analytic function, and (iii) these poles will be located in the lower half of the complex $\kappa$ plane and positioned symmetrically about the $\operatorname{Im}(\kappa)$ axis.
We want to find the positions and the residues of these poles in order to find the frequency, the damping, and the strength of the resonance. Let us sketch the process we employ to do this; it is similar to the process we used for the dc case: We first replace the impedance $Z$ by an analytic function $\bar{Z}$ that equals $Z$ on the real axis. In this case we obtain $\bar{Z}$ by replacing $|\kappa|$ by $\kappa$ and $\operatorname{sgn}(\kappa)$ by 1 in the expression for $\lambda$ [Eqs. (32),(33)]; we then insert this expression for $\lambda$ into Eq. (7). For this new function $\bar{Z}$ we now interpret $\kappa$ to represent a complex variable defined on the Riemann sheet $-\pi / 2<\theta<3 \pi / 2$. Once we have $\bar{Z}$ we obtain the position of its poles by finding the zeros of its denominator; unlike for the dc case we need to do this numerically. Given the poles we then find the residues. Figs. 6 and 7 display the results.

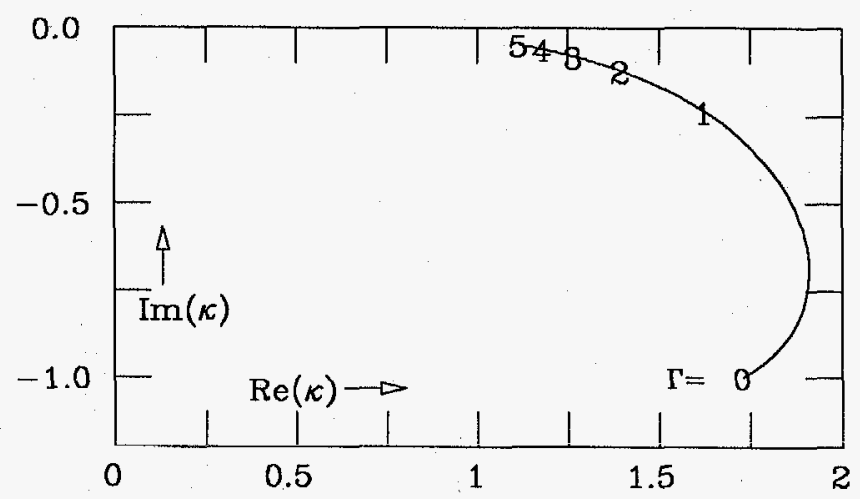

FIGURE 6. The movement in the complex $\kappa$ plane of the fourth quadrant pole of the impedance when $\Gamma$ varies from 0 to 5 . The symbols give the pole position for the integer values of $\Gamma$.

Fig. 6 shows the movement of the fourth quadrant pole of $\bar{Z}$ as $\Gamma$ varies from 0 to 5. The symbols give the pole position for integer values of $\Gamma$. Note that $\operatorname{Re}(\kappa)$ at the pole gives the resonant frequency $\kappa_{r}\left(=k_{r} s_{0}\right)$, while $-\operatorname{Im}(\kappa)$ gives the damping factor $\alpha_{r}\left(=a_{r} s_{0}\right)$. We see that after an initial increase the frequency decreases with increasing $\Gamma$ while the damping factor decreases monotonically. In Fig. 7 we show the results in another way. Fig. $7 \mathrm{a}$ and $7 \mathrm{~b}$ give, respectively, $\kappa_{r}$ and $\alpha_{r}$ as functions of $\Gamma$. At $\Gamma=0.4$, which corresponds to the $1 \mathrm{~cm}$ copper tube, we obtain $\kappa_{r}=1.87$ and $\alpha_{r}=0.52$, which are, respectively, an $8 \%$ increase and a $48 \%$ decrease over the dc results. The dashes in Fig. 7 represent the high $\Gamma$ approximations obtained earlier, $\kappa_{r}=(8 / \Gamma)^{1 / 4}$ and $\alpha_{r}=1 /(4 \Gamma)$. We note that for $\Gamma \gtrsim 1 / 2$ these approximations agree well with the exact results. Finally, in Fig. $7 \mathrm{c}$ we plot the amplitude of the resonator term $f_{r} \equiv-E_{z r}(0) b^{2} /(4 q)$ as function of $\Gamma$. 


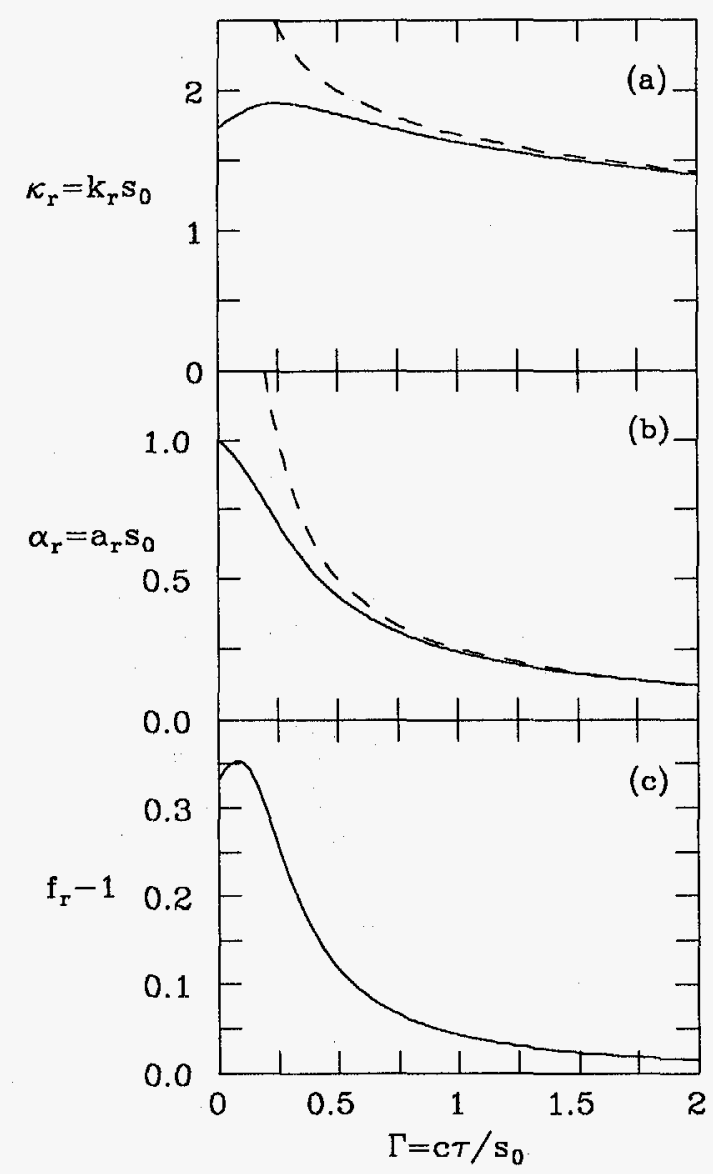

FIGURE 7. The resonant frequency $\kappa_{r}$, the damping factor $\alpha_{r}$, and the quantity $f_{r}-1$ as functions of $\Gamma$. The dashes represent the high $\Gamma$ approximations.

We have also found the total wakefield for the ac case by simply taking the inverse Fourier transform of $Z$ numerically. Fig. 8 displays the results when $\Gamma=0.4$, the case corresponding to a $1 \mathrm{~cm}$ copper tube. The dashed curve gives the resonator contribution. The dotted curve, for comparison, gives the total dc wake. We clearly see that the damping of the oscillation has decreased over the dc case. For this example the field changes sign 7 times; it is not until $s \approx 10 s_{0}$ that the field remains positive. We also note that the resonator approximates the short-range wake well.

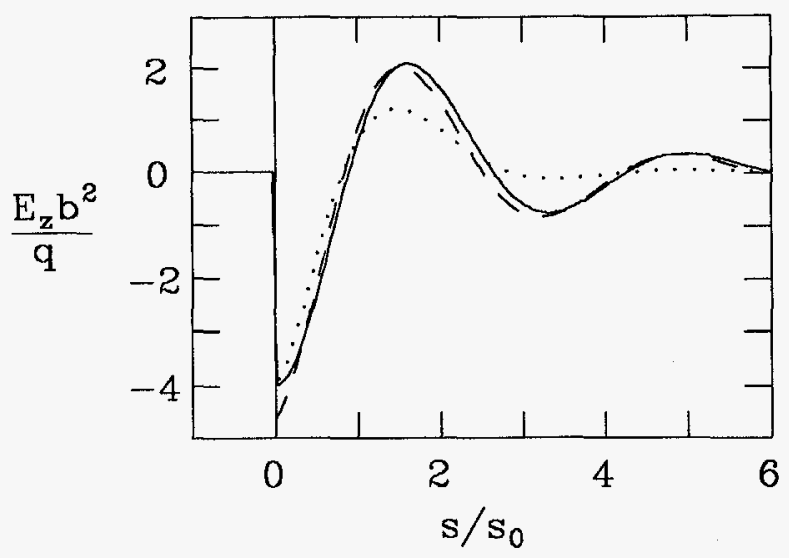

FIGURE 8. The longitudinal wakefield when $\Gamma=0.4$. The dashes display the resonator contribution. The dots give the dc results.

\section{THE WAKEFIELD OF A BUNCH DISTRIBUTION}

Once the impedance is known the wakefield for a charge distribution $E_{z b}$ can be obtained by performing the integration

$$
E_{z b}(s)=-\frac{q c}{2 \pi} \int_{-\infty}^{\infty} d k Z(k) \tilde{\lambda}_{z}(k) e^{-i k s},
$$

with $\tilde{\lambda}_{z}$ the Fourier transform of the longitudinal charge distribution $\lambda_{z}$. Piwinski analytically found the electric field induced by a long Gaussian bunch (long compared to $s_{0}$ ) in a resistive pipe with a constant conductivity (15). His method of solution was to take the impedance of Eq. (7), but keep only the first, low frequency term; then to multiply this by the Gaussian spectrum; and then to perform the inverse Fourier transform. His solution of the bunch field is

$$
E_{z b}=\frac{q}{4 b^{2}}\left(\frac{s_{0}}{\sigma_{z}}\right)^{3 / 2} f\left(s / \sigma_{z}\right)
$$

with

$$
f(u)=\left.|u|^{3 / 2} e^{-u^{2} / 4}\left(I_{1 / 4}-I_{-3 / 4} \mp I_{-1 / 4} \pm I_{3 / 4}\right)\right|_{u^{2} / 4} ;
$$

$q$ now represents the charge of the entire bunch and $\sigma_{z}$ is the bunch length In Eq. (36) the upper signs apply for $u<0$, the lower signs for $u>0$; the 
modified Bessel functions $I$ are evaluated at $u^{2} / 4$. Note that Eq. (36) reduces to Chao's result, Eq. (9), in the limit $\sigma_{z}$ approaches zero.

Following the same method, but using the impedance given by Eq. (8), we have numerically obtained the wakefield of a short Gaussian bunch in a tube with constant conductivity. The results, for $\sigma_{z} / s_{0}=0.2,0.5,1.0$, and 5.0 are shown in Fig. 9 (the dashed curves). These curves can represent the effect when for fixed geometry we increase $\sigma_{z}$ by a factor of 25 ; alternatively, since $s_{0}$ depends also on $b$, they also can represent the effect when for a fixed bunch length we decrease $b$ by a factor of 8.6. Note that by scaling the ordinate by $\left(\sigma_{z} / s_{0}\right)^{3 / 2}$ we obscure the fact that the interaction becomes stronger as the bunch length becomes shorter. For example, the peak value of $-E_{z b}$ is a factor of 63 times larger when $\sigma_{z} / s_{0}=0.2$ than it is when $\sigma_{z} / s_{0}=5.0$. Piwinski's result is nearly indistinguishable from the $\sigma_{z} / s_{0}=5.0$ curve; therefore, for $\sigma_{z} / s \gtrsim 5.0$ the long range result approximates the exact answer well.

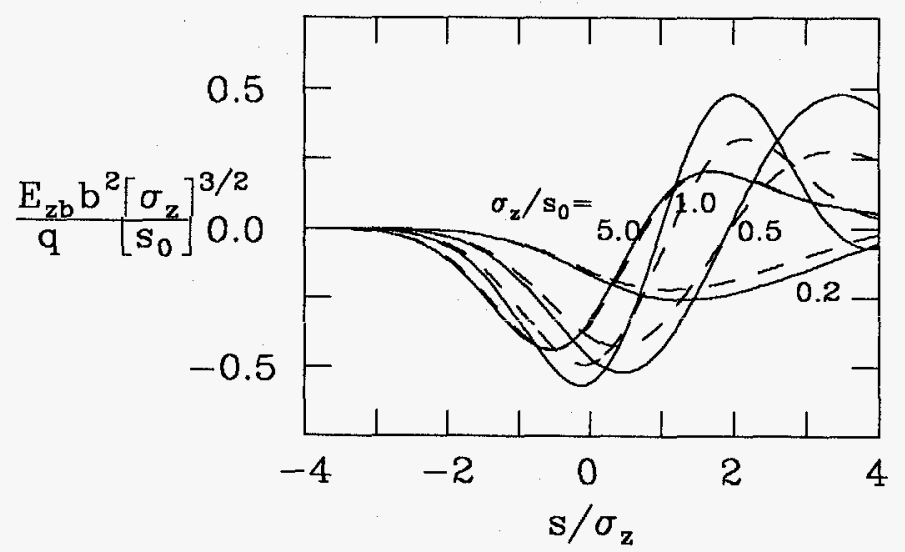

FIGURE 9. The longitudinal wakefield for a Gaussian bunch for a dc conductivity (the dashes) and for an ac conductivity, with $\Gamma=0.4$ (the solid curves). Curves are given for several values of $\sigma_{z} / s_{0}$.

We have repeated the calculations for an ac conductivity with $\Gamma=0.4$, the relaxation parameter for a $1 \mathrm{~cm}$ copper tube (the solid curves in Fig. 9). The ac curves represent the effect when, for a fixed geometry, we vary the bunch length. If we are considering a $1 \mathrm{~cm}$ copper tube, then the curves give the change in field when $\sigma_{z}$ varies from $4 \mu \mathrm{m}$ to $100 \mu \mathrm{m}$. Comparing the two sets of curves in the figure we see that the extrema are somewhat larger for the ac than for the dc curves. Note, however, that for the long bunch length, $\sigma_{z} / s_{0}=5.0$, there is little difference between the results of the two models.
An important parameter associated with the longitudinal wakefield is the loss factor $k_{t o t}$, which gives the total energy loss of a bunch due to the wakefield, divided by the bunch charge $q$. For a Gaussian bunch the loss factor is given by

$$
k_{\text {tot }}=\frac{c}{\pi} \int_{0}^{\infty} d k \operatorname{Re} Z(k) e^{-k^{2} \sigma_{z}^{2}} .
$$

We have computed $k_{t o t}$ for both the dc model and the ac model with $\Gamma=0.4$ (see Fig. 10). Note that at $\sigma_{z}=0$ the loss factor for both curves equals $E_{z}\left(0^{+}\right) / 2 q$, as is required from basic principles (2). The dashed curve in Fig. 10 gives, for comparison, the long bunch asymptotic value, obtained by averaging Piwinski's result [Eqs. (35),(36)]: $k_{t o t} \approx 0.2 / b^{2}\left(\sigma_{z} / s_{0}\right)^{-3 / 2}$.

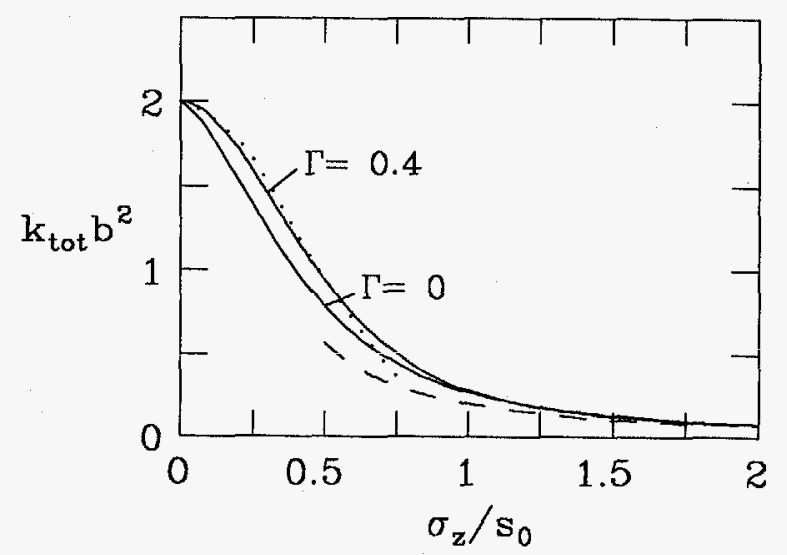

FIGURE 10. The loss factor for a short Gaussian bunch. Results are given for $\Gamma=0$ and for $\Gamma=0.4$. Also shown are the short bunch approximation $k_{\text {tot }}=$ $\left(2 / b^{2}\right) e^{-3 \sigma_{z}^{2} / s_{0}^{2}}$ (the dots) and the long bunch asymptotic result (the dashes).

From Fig. 10 we note that in calculating the loss factor for a short Gaussian bunch in a $1 \mathrm{~cm}$ copper tube we obtain only a slightly higher result when including the frequency dependence of the conductivity than when not including it; at maximum, near $\sigma_{z} / s_{0}=0.4$, the difference is $20 \%$. We also see that the loss factor is well approximated by the long range asymptote when $\sigma_{z} / s_{0} \gtrsim 1$. Finally, recall that for a dc conductivity the short range wake is dominated by a low $Q$ resonator with resonant frequency $\omega_{r}=\sqrt{3} c / s_{0}$. Taking as a rough model a resonator of this frequency, with no damping, and substituting into Eq. (37) we obtain $k_{t o t} \approx\left(2 / b^{2}\right) e^{-3 \sigma_{z}^{2} / s_{0}^{2}}$ (the dots). 


\section{ACKNOWLEDGEMENTS}

The authors thank S. Heifets for helpful discussions concerning the solution of integrals of functions of a complex variable.

\section{REFERENCES}

1. R. Tatchyn, et.al., "Research and Development Toward a 4.5-1.5 ̊̊ Linac Coherent Light Source (LCLS) at SLAC," contributed to the $17^{\text {th }}$ International Free Electron Laser Conf., New York, NY, Aug. 21-25, 1995.

2. Chao, A. W., Physics of Collective Beam Instabilities in High Energy Accelerators, New York, John Wiley \& Sons, Inc., 1993

3. Ashcroft, N., and Mermin, N., Solid State Physics, Orlando, Florida, Holt, Rinehart and Winston, Inc., 1976.

4. Reuter, G., and Sondheimer, E., Proc. Roy. Soc. A195, 336 (1949).

5. Courant, E. D., IEEE Trans. Nucl. Sci. NS-12, 550 (1965).

6. Laslett, L. J. and Sessler, A. M., in SLAC-49 (August 1965), p. 23.

7. Robinson, K. W., in SLAC-49 (August 1965), p. 32.

8. Dikansky, N. S., and Skrinsky, A. N., Atomnaya Energia 21, 176 (1966).

9. Morton, P. L., Neil, V. K., and Sessler, A. M., J. Appl. Phys. 37, 3875 (1966)

10. Henke, H., and Napoly, O., in Proc. of the $2^{\text {nd }}$ European Particle Accelerator Conference, Nice, France, Editions Frontièrs, 1990, p. 1046.

11. Henry, O., and Napoly, O., Part. Accel. 35, 235-248 (1991) and CERN-CEA, DPhN/STAS/91-R08 (May 1991).

12. Bane, K. L. F., SLAC-AP-87 (May 1991).

13. Jackson, J. D., Classical Electrodynamics, 2nd Edition, New York, John Wiley \& Sons, Inc., 1975.

14. Panofsky, W., and Wenzel, W., Rev. Sci. Instrum. 27, 967 (1956).

15. Piwinski, A., DESY Report $72 / 72$ (1972).

\section{DISCLAIMER}

This report was prepared as an account of work sponsored by an agency of the United States Government. Neither the United States Government nor any agency thereof, nor any of their employees, makes any warranty, express or implied, or assumes any legal liability or responsibility for the accuracy, completeness, or usefulness of any information, apparatus, product, or process disclosed, or represents that its use would not infringe privately owned rights. Reference herein to any specific commercial product, process, or service by trade name, trademark, manufacturer, or otherwise does not necessarily constitute or imply its endorsement, recommendation, or favoring by the United States Government or any agency thereof. The views and opinions of authors expressed herein do not necessarily state or reflect those of the United States Government or any agency thereof. 


\section{DISCLAMIER}

Portions of this document may be illegible in electronic image products. Images are produced from the best available original document. 